\title{
SPODi: Simulador do Perfil Operacional de Dispositivos Intravenosos para Auxilio à Tomada de Decisões Médicas
}

\author{
Fabrício Ferreira $^{1}$, Jorge Barbosa ${ }^{2}$, Felipe Gruendemann $^{1}$, \\ Roger Machado ${ }^{1}$, Adenauer Yamin ${ }^{1}$, Luciano Agostini ${ }^{1}$ \\ ${ }^{1}$ Universidade Federal de Pelotas (UFPel) - Pelotas - RS - Brasil \\ ${ }^{2}$ Universidade do Vale do Rio dos Sinos (Unisinos) - São Leopoldo - RS - Brasil \\ Email:\{fferreira,fcgruendemann,rdsmachado,adenauer,agostini\}@inf.ufpel.edu.br \\ jbarbosa@unisinos.br
}

\begin{abstract}
Infusion procedures are among the most common in hospitals and have a high volume of adverse events in their operation. This paper presents a simulator, called SPODi, that allows an agile construction of the operational profiles of intravenous devices, enabling them to be evaluated when adverse events occur during the period of use. SPODi simulator was validated with real data extracted from a physical prototype (SPODi_Med) which was specifically build with this function. The results obtained, considering a 95\% confidence interval when applying the $t$ Student statistical test, indicate that SPODi can be used to infer the behavior of real infusion equipment.
\end{abstract}

Resumo. Os procedimentos de infusão estão entre os mais usuais em hospitais e apresentam um volume elevado de eventos adversos na sua operação. Este artigo apresenta um simulador, denominado SPODi, que permite uma construção ágil dos perfis operacionais de dispositivos intravenosos, possibilitando que os mesmos possam ser avaliados quando da ocorrência de eventos adversos durante o período de seu uso. O simulador SPODi foi validado com dados reais extraídos de um protótipo físico (SPODi_Med) construído especificamente para essa finalidade. Os resultados obtidos, considerando um intervalo de confiança de $95 \%$ quando da aplicação do teste estatístico $t$ Student, apontam que o SPODi pode ser empregado para inferir o comportamento de um equipamento real de infusão.

\section{Introdução}

Os dispositivos médicos modernos, com a intenção de qualificar o esforço de gerência por parte dos profissionais da saúde, muitas vezes exploram operações autônomas nos diferentes procedimentos que realizam [Dey et al. 2018]. Entre os procedimentos clínicos mais rotineiros em hospitais, estão os tratamentos que empregam os sistemas intravenosos. Cerca de $80 \%$ dos pacientes hospitalizados carecem de recursos terapêuticos intravenosos [Cheng et al. 2016]. A entrega de medicamento intravenosa é prescrita considerando as ações das diferentes concentrações da absorção do medicamento na corrente sanguínea ao longo do tempo para diferentes métodos de entrega.

Os equipamentos de infusoterapia são utilizados em situações que exigem diferentes decisões por parte dos profissionais de saúde, as quais geram impactos diversos no tratamento do paciente. Assim, uma forma de contribuir para as decisões médicas consiste 
na disponibilização, em tempo integral, dos perfis operacionais das infusões intravenosas por meios computacionais [Oliveira dos Santos et al. 2018, Beaudoin et al. 2016].

Apesar de existirem diversos trabalhos na literatura sobre gerenciamento e monitoramento de sistemas intravenosos, nota-se que ainda são necessários esforços de pesquisa relacionados ao tema [Dey et al. 2018, Parker et al. 2017]. Por sua vez, em relação aos riscos relacionados ao uso de tecnologias na área da saúde, o órgão americano Emergency Care Research Institute - ECRI, que faz um ranqueamento anual dos dez principais perigos no ambiente hospitalar, identificou que os erros de infusão ficaram em primeiro lugar no ano de 2017 [ECRI 2017].

Este trabalho está focado na geração de soluções para redução de erros de infusão e apresenta um simulador intitulado SPODi (Simulador do Perfil Operacional de Dispositivos Intravenosos), o qual tem como principais contribuições: (i) o desenvolvimento de um modelo de procedimento de infusoterapia, ainda não disponível na literatura; (ii) o desenvolvimento de um simulador capaz de gerar conjuntos expressivos de dados que caracterize os diferentes momentos do procedimento de infusão, sem necessidade de coletas de dados reais; (iii) o desenvolvimento de uma plataforma em hardware e firmware para a coleta de dados reais para a validação do simulador SPODi.

O desenvolvimento do simulador SPODi, tem por finalidade viabilizar a realização de diferentes avaliações, já que as infusões reais requerem longos períodos de tempo. Tipicamente, a duração de um único ensaio de escoamento de líquidos medicamentosos são superiores a horas. As taxas de escoamentos das infusões podem ser dosadas entre uma faixa de $(0,1$ a 999,9) $\mathrm{mL} / \mathrm{h}$ com reajustes da dosagem de $0,1 \mathrm{~mL} / \mathrm{h}$, apresentam alternâncias nos volumes infundidos e são, habitualmente, prescritos em reservatórios de medicamentos entre os limites de (50 a 10000) $m L$ [NBR 60601-2-24 2015]. Desta forma, o tempo envolvido na aquisição dos dados, com o potencial de abranger todas as configurações possíveis dos tratamentos de infusoterapia, dificultam a realização de pesquisas na área. Assim, uma das formas de evitar esse inconveniente é a exploração de simuladores de software-hardware [Ziuzev et al. 2014].

O simulador SPODi foi validado através de uma prototipagem funcional e testes estatísticos, sendo comparados os conjuntos de dados coletados pela plataforma desenvolvida, com os dados gerados pelo simulador.

Os testes realizados se mostraram promissores e apontam para a continuidade da pesquisa do simulador (SPODi) desenvolvido, o qual poderá ser usado na concepção de novas abordagens para aquisição e tratamento de informações oriundas de sistemas para infusão intravenosa.

O restante do texto está organizado nas seguintes seções. A Seção 2 traz uma revisão de trabalhos relacionados; a Seção 3 discute o desenvolvimento do simulador SPODi; a Seção 4 descreve o processo de validação do simulador SPODi; a Seção 5 apresenta os resultados e discussões; por fim, a Seção 6 conclui o artigo.

\section{Trabalhos Relacionados}

Para busca de trabalhos relacionados, foi realizada uma revisão nas bases Pubmed, Cochrane e Scielo. Estas bases, segundo a literatura, são entendidas como representativas na área [Oliveira dos Santos et al. 2018, Berdot et al. 2013]. 
Usando para busca em janeiro de 2019, os termos "simulation" AND "delivery" AND "intravenous" AND "drugs", abrangendo os anos entre 2009 a 2019, chegou-se a um total de 38 artigos. De modo que, após uma leitura dos textos, destaca-se os seguintes temas das publicações: (i) implementações de modelos de farmacocinética, em um total de nove artigos; (ii) avaliações da qualidade dos tratamentos por vias endovenosas, com seis publicações; (iii) discussões sobre os erros de medicações no decorrer do tratamentos clínicos, com três estudos publicados. Apenas dois trabalhos se mostraram de interesse para a pesquisa apresentada nesse artigo, pois são os trabalhos que tratam diretamente da entrega de medicamentos intravenosos. Os termos de buscas relacionados a entrega de medicamentos por sistemas de infusões intravenosos unidos ao simulador resultaram em uma baixa incidência de trabalhos disponíveis na literatura. Porém, há relatos atuais de pesquisadores que apresentam elevada expectativa por trabalhos futuros sobre o tema com foco em melhorias em sistemas intravenosos capazes de auxiliar as ações de tomadas de decisões médicas [Piper et al. 2018, Yu et al. 2017, Blandford et al. 2016].

O trabalho de [Pierce et al. 2013] conduziu uma simulação do comportamento da vazão "livre", com a pinça rolete do equipo (tubo construído com polímero, pelo qual ocorre a infusão) totalmente aberta. A análise é baseada na medição dos volumes entregues por micro-gotejamento influenciado pelo efeito da força da gravidade, empregando uso de três conjuntos de equipos de fabricantes distintos. Com o resultado da simulação, foi possível identificar variações no tempo total da duração das infusões alterando entre 2,9 vezes o escoamento dos líquidos em testes. A análise dos resultados obtidos foi baseada pelos cálculos da variância não paramétrica no teste estatístico com um intervalo de confiança de $95 \%$. Além disso, os autores caracterizam que em uma vazão "livre" a resistência é variável e implica em regimento de escoamento não laminar para o modelo micro-gotas, de modo a dificultar a formulação matemática do modelo do escoamento.

No artigo de [Murphy and Wilcox 2010] é descrito um modelo matemático de um sistema de infusão usado para investigar e verificar um cenário de falhas em múltiplas bombas de infusões, o qual pode ser aplicado para determinar a taxa de escoamento dos sistemas intravenosos. De acordo com os autores, a tecnologia de Bomba de Infusão de Seringa, tem o comportamento da taxa de escoamento de acordo com a lei de Poiseuille, a qual define o escoamento causado por um gradiente de pressão, com sua velocidade de vazão caracterizado pela resistência física dos componentes (linha de infusão, cateter e cânula). Esse modelo empregou as equações Poiseuille, com uso do software do MATrix LABoratory - MATLAB com aplicação da ferramenta Simulink. O estudo introduziu uma simulação matemática de um sistema de bombeamento para examinar várias questões operacionais práticas. Seus resultados indicam que a degradação da taxa de escoamento do líquido medicamentoso é atribuível a mudanças no volume extracorporal do sistema de infusão.

Esses dois trabalhos relacionados apresentam características que contribuíram com o desenvolvimento do simulador SPODi, incluindo o emprego de métodos de avaliação por testes estatísticos e o uso do MATLAB como uma ferramenta de desenvolvimento.

\section{Visão Geral do Simulador SPODi}

As funcionalidades do simulador SPODi foram concebidas para possibilitar a geração das principais taxas de escoamentos intravenosos. O objetivo principal do SPODi é permitir 
a captura de dados de diferentes perfis de infusão (contemplando variação na velocidade de escoamento, na quantidade do volume infundido e na duração do tempo das infusões), em um tempo bastante inferior ao necessário para a coleta de dados de uma infusão real.

Os principais aspectos implementados no simulador SPODi são: (i) modelagem matemática que define o comportamento da célula de carga; (ii) modelagem matemática que rege os escoamentos de líquidos por efeito da gravidade; (iii) especificações físicas dos componentes da linha de infusão (equipo e reservatório de medicamento); (iv) operações de acordo com os requisitos normativos vigentes para ensaios de dispositivos de infusões; e (v) validações dos dados com os parâmetros de tolerâncias aceitáveis.

O simulador SPODi, foi desenvolvido em uma aplicação no software MATLAB R2018a, o qual possibilitou a formulação matemática das representações dos elementos que compõem o sistema de infusão intravenoso. Assim, buscou-se um conjunto de parâmetros que permita explorar o modelo empregado. Na sequência são listados os principais conteúdos e métricas que definiram as especificações do simulador:

1. Fenômeno de Transporte: obtenção das fórmulas para o calculo da velocidade do escoamento de líquidos em queda livre [Trigueiro and Lira 2014];

2. Modelo da Célula de Carga: descrição matemática do comportamento do sinal elétrico do sensor Strain Gauges [Kleckers 2018];

3. Norma Técnica: definições dos requisitos de configurações dos ensaios de infusão, sendo estipulado altura do reservatório de medicamento $h=1 \mathrm{~m}$, densidade do líquido infusão $\rho=0,998 \mathrm{~g} / \mathrm{mL}$, exatidão da quantidade do líquido entregue $E= \pm 5 \%$, relação da incidência de 20 gotas com a equivalência de $1 \mathrm{~mL}$ e os limites de validade dos equipos entre 24 a 96 horas [NBR 60601-2-24 2015, NBR 8536-5 2012];

4. Agência Fiscalizadora: especificações das dimensões e os pesos das embalagens dos reservatórios de medicamentos relacionados aos seus volumes nominais [MELLO 2010].

\subsection{Modelagem Matemática do Simulador SPODi}

A modelagem do simulador SPODi é descrita na sequência, incluindo as especificações das equações matemáticas e do formalismo do comportamento para simulação. As equações que regem o comportamento da quantidade do volume entregue são apresentadas em (1), sendo definidas por: (1) a) $\left[v\left(\frac{m}{s}\right)\right]$ velocidade de escoamento do líquido de infusão, $[g]$ aceleração da gravidade, $[\Delta . h]$ diferença de altura do líquido; (1) b) $\left[Q\left(\frac{m^{3}}{s}\right)\right]$ vazão de escoamento, $\left[a_{\text {equipo }}\right]$ área interna de abertura no equipo, ajustada pela pinça rolete; (1) c) [ $\left.V_{\text {entregue }} m L\right]$ a quantidade de volume entregue, [i] iésima amostra da quantidade de volume entregue, $[n]$ última amostra de quantidade de volume e $[\Delta . t]$ diferença de tempo.

$$
\begin{array}{lll}
\text { a) } v=\sqrt{2 . g \cdot \Delta h} & \text { b) } Q=a_{\text {equipo }} . v & \text { c) } V_{\text {entregue }}=\sum_{i=1}^{n} V_{\text {entregue }}+Q . \Delta . t
\end{array}
$$

Assim, as equações empregadas no simulador que representam a definição do comportamento do sinal elétrico do sensor célula de carga, Strain Gauge, são descritas em (2) [Kleckers 2018]. 


$$
\text { a) } V_{\text {saida }}=V c c . C \cdot \frac{F_{\text {peso }}}{F_{\text {nominal }}} \quad \text { b) } F_{\text {peso }}=V_{\text {entregue }} \rho \cdot \frac{\left(d_{1}-d_{2}\right)}{d_{2}}
$$

As variáveis das equações em (2) a), são: $\left[V_{\text {saida }}(\right.$ Volts $\left.)\right]$ tensão elétrica de saída do sensor, $[V c c]$ tensão elétrica de alimentação na célula de carga, $[C]$ sensibilidade nominal do Strain Gauge, $\left[F_{\text {peso }}\right]$ força aplicada sobre o ponto de medição do sensor e $\left[F_{\text {nominal }}\right]$ força nominal de leitura estipulada pelo fabricante do sensor. Já em (2) b) são: $[\rho]$ densidade do líquido medicamentoso, $\left[d_{1}\right]$ comprimento da haste suporte que é a distância do ponto de articulação e fixação do medicamento e $\left[d_{2}\right]$ posição da aplicação da força que é a distância do ponto de articulação da haste de suporte e contado do Strain Gauge.

\subsection{Implementação do Simulador SPODi}

O software MATLAB foi utilizado no desenvolvimento do SPODi, empregando a ferramenta Graphical User Interface Development Environment - GUIDE, a qual permitiu a construção de uma interface de manipulação dos dados sobre as infusões com a vinculação das aplicações das equações que caracterizam o sistema intravenoso.

Como exemplo de uso do simulador SPODi, a Figura 1 a) apresenta a tela de configuração do simulador concebido, com os valores especificados para uma infusão. A Figura 1 b) exibe os gráficos dessa simulação representando o comportamento do perfil da infusão gravitacional.
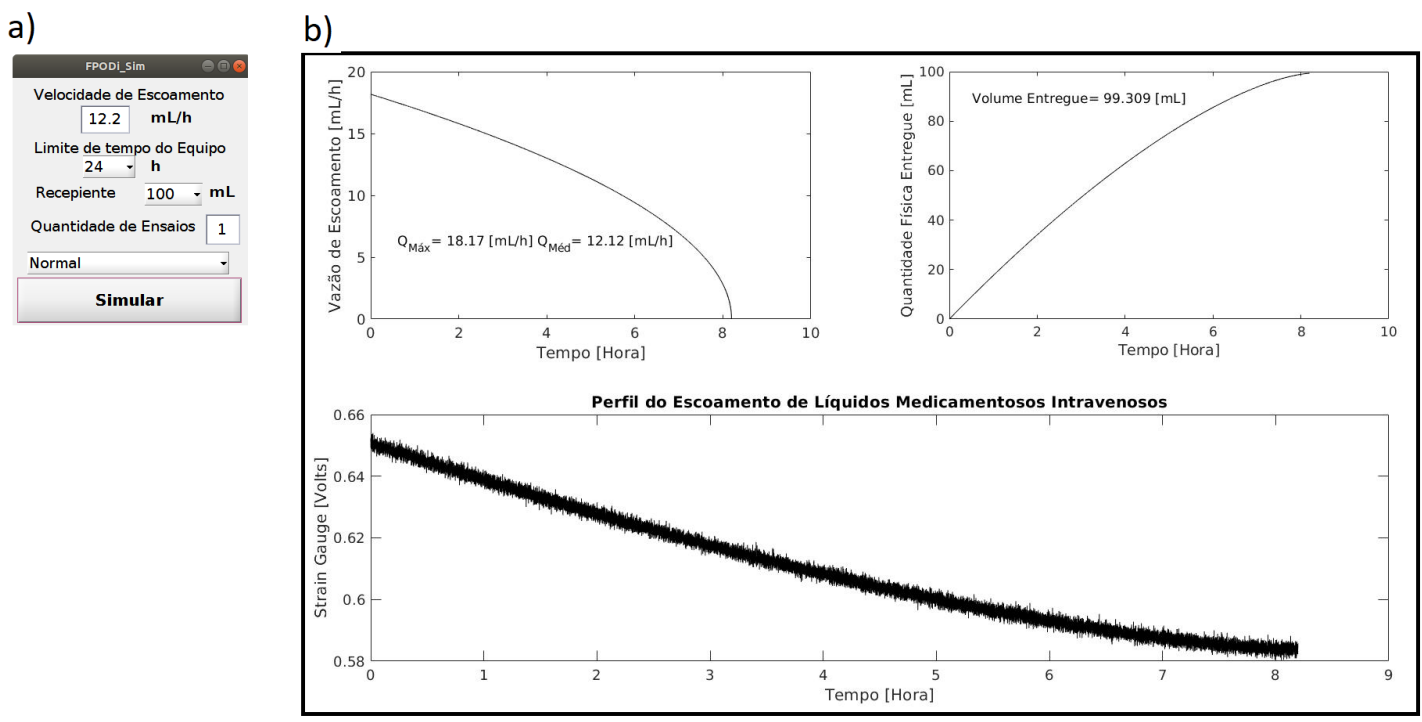

Figura 1. Dados de Entrada e Saída do SPODi.

Os requisitos normativos vigentes foram usados como parâmetros de validação da simulação. Estes requisitos definem as tolerâncias aceitáveis para o comportamento das vazões intravenosas [NBR 60601-2-24 2015]. A inserção do valor de tolerância considerou toda a gama dos limites estabelecidos. A escolha do valor da tolerância aplicada foi individual para cada ensaio e tendo o processo de seleção por sorteios randômicos, 
pseudo-aleatórios, de modo a possibilitar uma simulação capaz de varrer as grandezas físicas toleráveis dos processos de fabricação.

Assim, os critérios de aceite para cada conjunto de dados simulados atendem às limitações existentes no mundo físico, possibilitando varrer as amplas variações das amplitudes existentes em coletas reais e sendo capaz de gerar um conjunto de dados simulados com características distintas.

\section{Validação do Simulador SPODi}

Na revisão da literatura desenvolvida neste trabalho, não foram encontrados trabalhos que apresentem simuladores de sistemas de infusões intravenosas. Assim, foi impossível a comparação com resultados de outros simuladores na validação do SPODi. Deste modo, para a validação do simulador SPODi foi necessária a construção de um sistema físico, denominado SPODi_Med, capaz de efetuar as medições dos perfis das infusões reais.

OSPODi_Med, foi concebido considerando os conjuntos de requisitos normativos vigentes, os quais especificam conjuntos de regras dos comportamentos funcionais para as características físicas pertinentes as infusões intravenosas [NBR 60601-2-24 2015].

A concepção do SPODi_Med, enquanto sistema de medição de dados, tem como premissa a coleta da quantidade de líquido infundido, introduzindo um método de sensoriamento na linha de infusão. A Figura 2 a) apresenta o projeto mecânico com as principais especificações dimensionais, bem como a linha de infusão, formada por um equipo associada ao reservatório de medicamento. A Figura 2 b) mostra a imagem real do SPODi_Med e também o hardware e firmware que compõem o sistema supervisório concebido para o mesmo.

De forma mais específica, o SPODi_Med foi desenvolvido em três frentes: (i) a construção de sistema mecânico capaz de obter a medição contínua da quantidade de líquidos a serem infundidos; (ii) o desenvolvimento de sistema eletrônico apropriado para aquisições, pré-processamento e registro dos sinais elétricos que caracterizam a entrega de líquidos de medicamentos intravenosos; (iii) a programação do firmware para a criação de um sistema supervisório propício ao gerenciamento do hardware e sistema eletromecânico. Na sequência são apresentadas essas três etapas.

\subsection{Sistema Mecânico do SPODi_Med}

A constituição do sistema mecânico do SPODi_Med foi feita empregando uma estrutura de tripé de fotografia, como base de fixação dos componentes do sensor e os descartáveis hospitalares (equipo e reservatório). A principal função do sistema mecânico é garantir a fixação do elemento do sensor sem ocorrência de deformações mecânicas na sua estrutura devido à aplicação da força peso do líquido contido no reservatório de medicamento, que pode variar o seu peso de acordo com o conteúdo da embalagem (tipicamente os volumes nominais mais empregados são: $50 \mathrm{~mL}, 100 \mathrm{~mL}, 250 \mathrm{~mL}, 500 \mathrm{~mL}$ e $1 L$ [Bhavasaar et al. 2016]).

O material empregado na haste do elemento do sensor foi o alumínio, pois é um material com baixa densidade e, assim, com pouco peso. Por outro lado, no ponto de apoio, que é o local do sensor que recebe ação da força peso, foi aplicada uma região de aço inoxidável, que é um material com maior densidade e, assim, apresenta uma maior 

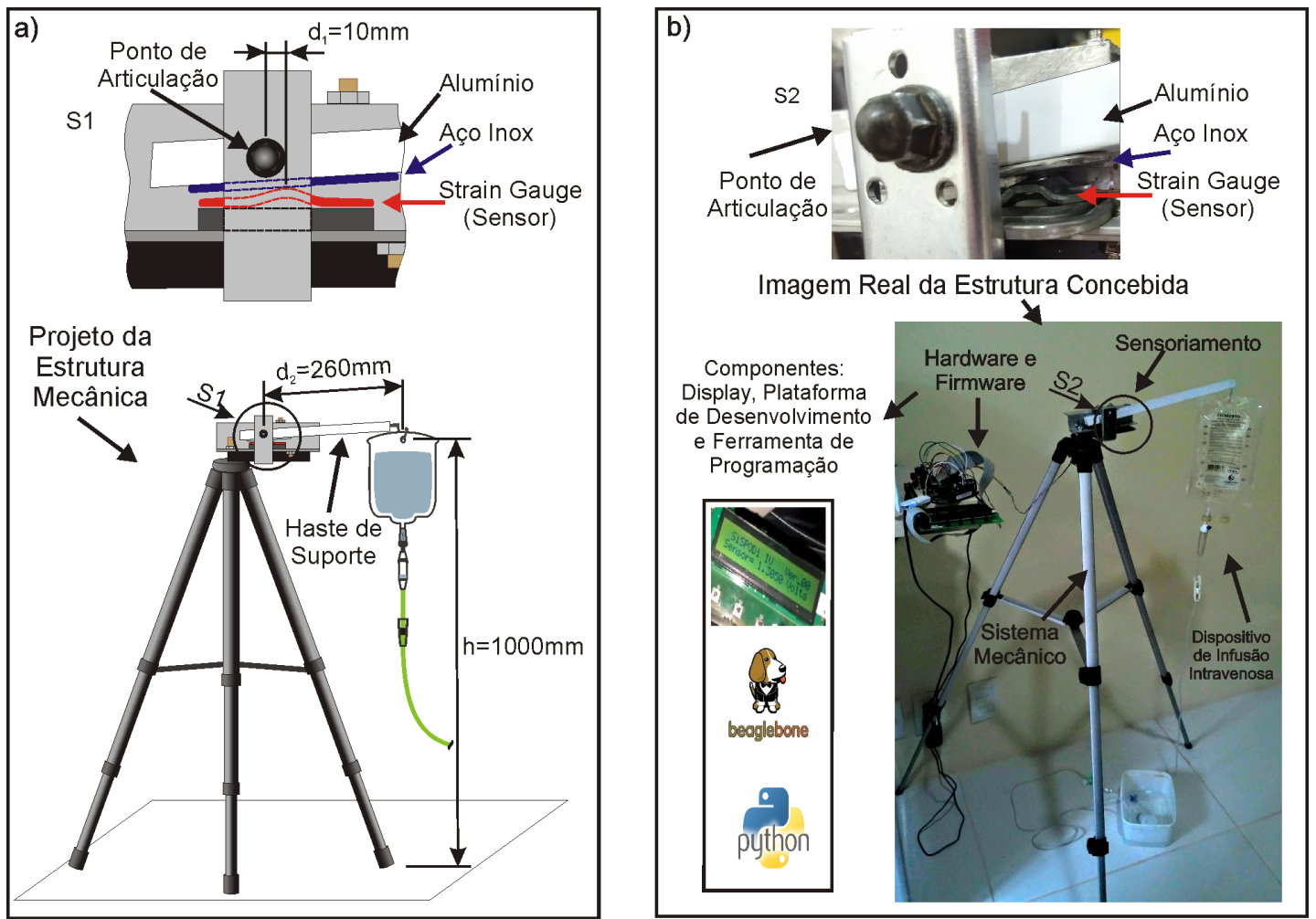

Figura 2. Detalhamento do Projeto SPODi_Med.

resistência à compressão sem se deformar. As Figuras 2 a) e 2 b) ilustram as seções S1 e $\mathrm{S} 2$ com os elementos do sensor (alumínio e aço inox) e os pontos de contatos sobre o transdutor.

A leitura da força peso dos componentes do sistema intravenoso, está diretamente relacionada com os volumes presentes no reservatório de medicamento, logo, as suas massas podem variar entre uma faixa aproximada de $0 \mathrm{~g}$ a $1 \mathrm{Kg}$. Desta forma, a determinação da força peso envolve os seguintes cálculos: (i) aceleração da gravidade, que tem seu valor de constante, usualmente, igual à $g=9,8 \mathrm{~m} / \mathrm{s}^{2}$; (ii) cálculo da força peso, definida pela equação (3) a); e (iii) força aplicada no sensor no ponto de ancoragem, definida pelo momento de força em relação aos pontos de apoio e a articulação. Assim, o equacionamento da força de compressão, Força Peso $-F P$, sobre o ponto de contato do sensor é determinada pela equação (3) b).

$$
\text { a) } P=m . g \quad \text { b) } F P(N)=\frac{\left(d_{2}-d_{1}\right) \cdot P}{d_{1}}
$$

Essas equações são definidas por: (3) a) $[P]$ peso, $[m]$ massa e $[g]$ aceleração da gravidade; (3) b) $[F P]$ força peso, $[d 2]$ comprimento da haste até ponto de articulação, $[d 1]$ distância da articulação até o ponto de contato no sensor. Na Figura 2 a) são identificadas as distâncias $d 1$ e $d 2$.

\subsection{Sistema Eletrônico do SPODi_Med}

O SPODi_Med usa um extensômetro (sensor Strain Gauge com descrição de código CZL902) para medir a força peso no ponto de ancoragem e, assim, gerar os dados de vo- 
lume escoado, a partir do qual todos os demais dados são inferidos [Sea Electronic 2019]. O transdutor CZL902, gerou dados equivalentes ao sensor simulado. Os sensores Strain Gauge alteram suas resistências elétricas a partir do peso que recebem. Assim, é possível produzir estímulos elétricos que, devidamente caracterizados, podem ser processados e manipulados por sistemas computacionais. A configuração das ligações elétricas que envolvem aplicações de Strain Gauge, normalmente são circuitos baseados em pontes de Wheatstone que permitem obter uma leitura com precisão mesmo com sinais elétricos com baixa amplitude [Hottinger 2018].

O sistema eletrônico do SPODi_Med foi subdividido em duas partes, onde na primeira foi utilizada uma placa microprocessada comercial, apta a embarcar um sistema operacional open source Linux. A placa Beaglebone Black Rev-C da empresa Texas Instruments foi escolhida por possuir os recursos necessários para implementar o sistema. Já na segunda parte foi desenvolvido um circuito eletrônico específico com as funções de pré-processamento dos sinais elétricos obtidos pelo sensor Strain Gauge e de ligação elétrica do sensor com a placa microprocessada (Beaglebone).

Na Figura 3 a), é apresentada a imagem do sistema microprocessado empregado no protótipo, enquanto que na 3 b) são ilustrados os blocos do esquema elétrico, o qual é dividido em: (i) circuito de ligação do sensor de força peso, Strain Gauge; (ii) amplificação diferencial do sinal elétrico com ganho de sinal de $A=300$; (iii) etapa de pré-processamento, filtro passa-baixa com frequência de corte $F C=6 \mathrm{~Hz}$. Por fim, a 3c mostra a imagem do sensor Strain Gauges e a interface de display alfanumérico.
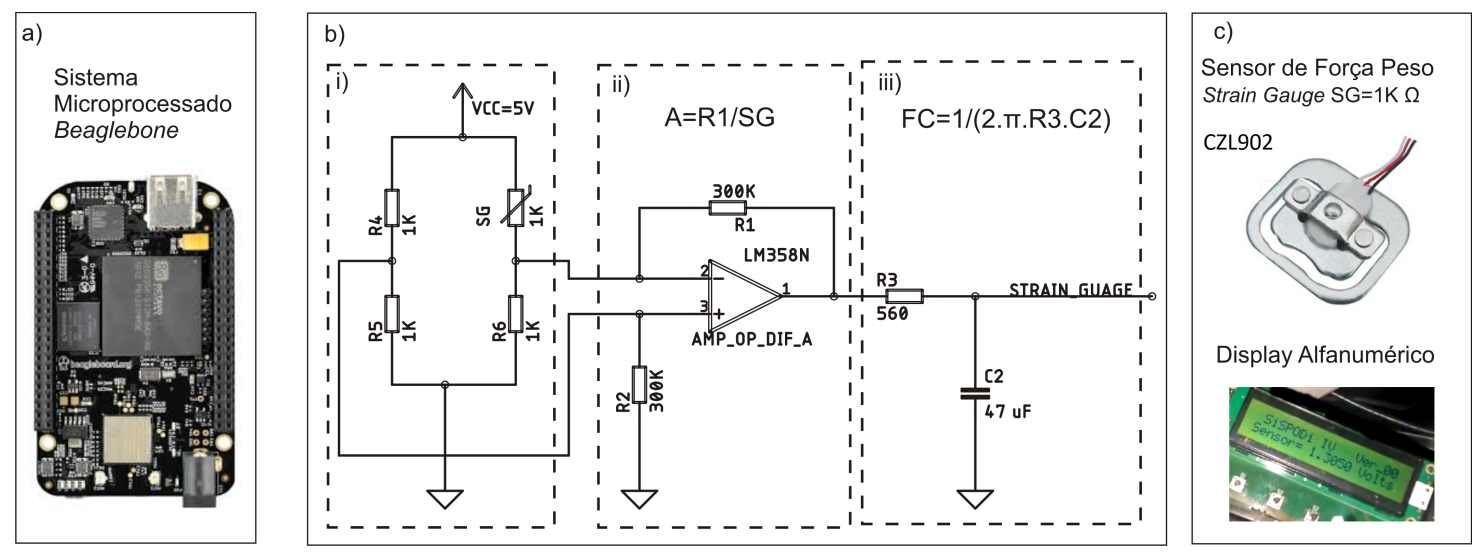

Figura 3. Hardware do SPODi_Med.

\subsection{Programação do Firmware do SPODi_Med}

O firmware do SPODi_Med foi desenvolvido como uma aplicação embarcada em Linux. O ambiente de desenvolvimento empregado foi a ferramenta Spyder 3.2.6, que é uma plataforma integrada open source para programação científica na linguagem Python.

As responsabilidades do firmware são: (i) configurar o hardware da Beaglebone, realizar o setup do conversor analógico digital e apresentar as instruções da interface do display alfanumérico; (ii) gerenciar o armazenamento dos dados; (iii) sincronizar os eventos no tempo. 


\section{Resultados}

A comprovação da validade dos conjuntos de dados sobre as infusões, experimentais e simulados, foi realizada com base na análise de confronto de igualdade, com o emprego das técnicas de testes estatísticos denominado $t$ Student. A Figura 4, ilustra o exemplo de uma infusão gravitacional, sendo que na Figura 4 a) são mostrados os gráficos dos conjuntos de dados elétricos experimental e simulado para uma taxa de escoamento de $12,2 \mathrm{~mL} / \mathrm{h}$. Enquanto que a Figura $4 \mathrm{~b}$ ), mostra graficamente os resultados dos cálculos das médias e desvios padrões, de modo a possibilitar uma análise da analogia entre seus dados.
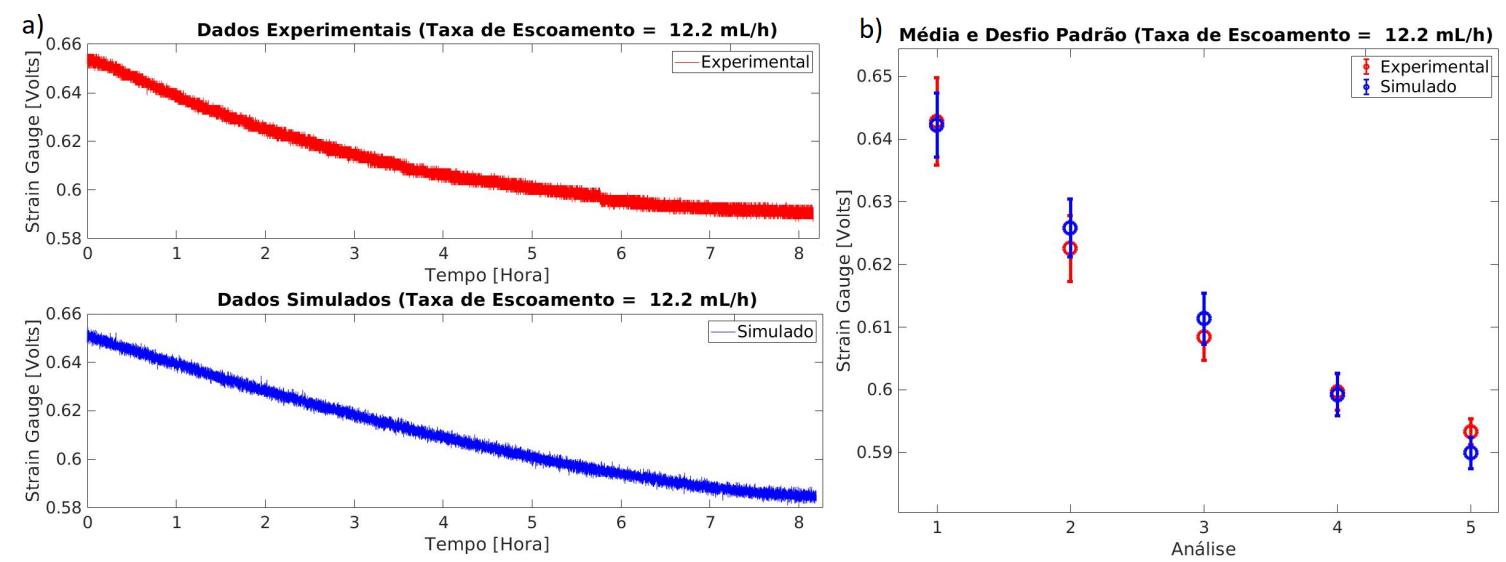

Figura 4. Comparação dos Dados do SPODi_Med e SPODi.

$\mathrm{Na}$ etapa de aquisição dos valores reais por meio da plataforma projetada, foram escolhidas configurações que têm o potencial de representar a entrega de medicamento intravenoso por gravidade. No total, foram ensaiados sete tipos de vazões com os tempos de duração das infusões diferentes e três quantidades de volumes entregues. Após a obtenção dos dados experimentais, foi utilizado o simulador desenvolvido para gerar os valores paras as mesmas velocidades de escoamento e quantidade de líquido entregue.

Na Tabela 1 são apresentadas as vazões ensaiadas, a duração do ensaio, a quantidade física do volume infundido e a análise do conjunto de dados elétricos, os quais foram subdivididos em dados experimentais (SPODi_Med) e dados simulados (SPODi), com os cálculos: (i) média; (ii) desvio padrão; (iii) desvio relativo; (iv) diferenças das médias dos sensores experimental e simulado. A análise estatística usou uma janela temporal de 1 hora e 30 minutos, que caracteriza o período de tempo necessário para estabilizar a vazão [NBR 60601-2-24 2015], sendo que o tempo total de todos os ensaios é de 38 horas e 42 minutos.

Na comparação das duas populações amostrais, experimental (SPODi_Med) e simulado (SPODi), foi aplicado o Teste de Hipótese para Duas Amostras Dependentes ( $t$ pareado), que é um teste paramétrico e tem por característica o confronto da igualdade das medições efetuadas em tempos diferentes.

A aplicação do teste de igualdade envolve o cálculo das médias, das diferenças das médias e o desvio padrão das diferenças das médias. Esses valores estão apresentados na Tabela 2. Logo, os resultados apresentados nas Tabelas 1 e 2, permitem observar que as diferenças entre as médias são próximas de zero, caracterizando sua equivalência. 
Tabela 1. Valores dos Dados Obtidos SPODi_Med e SPODi.

\begin{tabular}{|c|c|c|c|c|c|c|c|c|}
\hline \multirow{2}{*}{$\begin{array}{l}\text { Vazão } \\
\left(\frac{m L}{h}\right)\end{array}$} & \multirow{2}{*}{$\begin{array}{l}\text { Duração } \\
\text { Ensaios } \\
(h: m)\end{array}$} & \multirow{2}{*}{$\begin{array}{l}\text { Volume } \\
\text { Entregue } \\
(m L)\end{array}$} & \multicolumn{2}{|c|}{ SPODi_Med $(V)$} & \multicolumn{2}{|c|}{ SPODi $(V)$} & \multirow{2}{*}{$\begin{array}{l}\text { Desvio } \\
\text { Relativo } \\
\text { (\%) }\end{array}$} & \multirow{2}{*}{$\begin{array}{l}\text { Diferenças } \\
\text { das médias } \\
(V)\end{array}$} \\
\hline & & & Média & $\begin{array}{l}\text { Desvio } \\
\text { Padrão }\end{array}$ & Média & $\begin{array}{l}\text { Desvio } \\
\text { Padrão }\end{array}$ & & \\
\hline \multirow{3}{*}{5,0} & \multirow{3}{*}{ 05:00 } & \multirow{3}{*}{25} & 0,7410 & 0,0034 & 0,7371 & 0,0027 & 0,5263 & 0,0039 \\
\hline & & & 0,7337 & 0,0037 & 0,7307 & 0,0024 & 0,4089 & 0,0030 \\
\hline & & & 0,7268 & 0,0034 & 0,7260 & 0,0020 & 0,1101 & 0,0008 \\
\hline \multirow{3}{*}{8,7} & \multirow{3}{*}{ 05:07 } & \multirow{3}{*}{45} & 0,7156 & 0,0051 & 0,7155 & 0,0036 & 0,0140 & 0,0001 \\
\hline & & & 0,7036 & 0,0025 & 0,7048 & 0,0030 & 0,1706 & $-0,0012$ \\
\hline & & & 0,6987 & 0,0019 & 0,6966 & 0,0023 & 0,3006 & 0,0021 \\
\hline \multirow{5}{*}{12,2} & \multirow{5}{*}{ 08:09 } & \multirow{5}{*}{100} & 0,6428 & 0,0069 & 0,6418 & 0,0051 & 0,1556 & 0,0010 \\
\hline & & & 0,6225 & 0,0052 & 0,6254 & 0,0046 & 0,4659 & $-0,0029$ \\
\hline & & & 0,6084 & 0,0037 & 0,6109 & 0,0040 & 0,4109 & $-0,0025$ \\
\hline & & & 0,5997 & 0,0029 & 0,5987 & 0,0034 & 0,1668 & 0,0010 \\
\hline & & & 0,5933 & 0,0021 & 0,5894 & 0,0025 & 0,6573 & 0,0039 \\
\hline \multirow{4}{*}{16,0} & \multirow{4}{*}{$06: 17$} & \multirow{4}{*}{100} & 0,6631 & 0,0097 & 0,6662 & 0,0067 & 0,4675 & $-0,0031$ \\
\hline & & & 0,6341 & 0,0062 & 0,6450 & 0,0058 & 1,7190 & $-0,0109$ \\
\hline & & & 0,6233 & 0,0026 & 0,6275 & 0,0047 & 0,6738 & $-0,0042$ \\
\hline & & & 0,6184 & 0,0022 & 0,6147 & 0,0031 & 0,5983 & 0,0037 \\
\hline \multirow{3}{*}{16,9} & \multirow{3}{*}{$05: 54$} & \multirow{3}{*}{100} & 0,7047 & 0,0117 & 0,7128 & 0,0071 & 1,1494 & $-0,0081$ \\
\hline & & & 0,6793 & 0,0057 & 0,6907 & 0,0060 & 1,6782 & $-0,0114$ \\
\hline & & & 0,6658 & 0,0035 & 0,6727 & 0,0047 & 1,0363 & $-0,0069$ \\
\hline \multirow{3}{*}{18,2} & \multirow{3}{*}{$05: 28$} & \multirow{3}{*}{100} & 0,6944 & 0,0105 & 0,6967 & 0,0072 & 0,3312 & $-0,0023$ \\
\hline & & & 0,6699 & 0,0050 & 0,6742 & 0,0060 & 0,6419 & $-0,0043$ \\
\hline & & & 0,6567 & 0,0034 & 0,6565 & 0,0044 & 0,0305 & 0,0002 \\
\hline 35,0 & $02: 50$ & 100 & 0,7180 & 0,0154 & 0,7206 & 0,0127 & 0,3621 & $-0,0026$ \\
\hline
\end{tabular}

Tabela 2. Média e Desvio Padrão das Diferenças (SPODi_Med e SPODi).

\begin{tabular}{c|c}
\hline Média das Diferenças & $-0,0019(V)$ \\
\hline Desvio Padrão das Diferenças & $0,0045(V)$ \\
\hline
\end{tabular}

Para realizar o teste $t$-pareado foi definida a hipótese de que a diferença entre as médias seja igual a zero, $\mu D=0$, com um intervalo de confiança- IC de $I C(95 \%)$. O teste bilateral é definido pelas equações (4), onde as suas variáveis são descritas: (4) a) $\left[T_{o b s}\right]$ Teste $t$ Observável, $[\bar{D}]$ médias das diferenças das médias, $[\mu D]$ média da diferença, $\left[S_{D}\right]$ desvio padrão das diferenças das médias e $[n]$ números de médias participantes; (4) b) $\left[t_{(n-1)}\right]$ grau de liberdade da distribuição $t$ Student e $[I C]$ intervalo de confiança; (4) c) limites para ser aceita a hipótese de igualdades dos sensores.

$$
\text { a) } T_{o b s}=\frac{\bar{D}-\mu D}{\frac{S_{D}}{\sqrt{n}}} \text { b) } t_{(n-1)}: I C(95 \%)=2,086 \text { c) } T_{\text {obs }}>2,086 \text { ou } T_{\text {obs }}<-2,086
$$

Assim, o valor calculado de $T_{o b s}=-1,93$ logo, está de acordo com o limite estabelecido (4) c), de modo a caracterizar o aceite da hipótese do teste $t$ Student. 


\section{Conclusões}

Este artigo apresentou o simulador SPODi, que tem como principais contribuições o desenvolvimento de um modelo de procedimento de infusoterapia, a concepção de um simulador para esse modelo e o projeto de uma plataforma para a coleta de dados reais para a validação do simulador.

As avaliações apresentadas no artigo permitiram concluir que as médias dos dados experimentais que são coletados pela plataforma desenvolvida e o conjunto de dados simulados são equivalentes. Essa equivalência foi comprovada com base no teste $t$ Student com um intervalo de confiança de $95 \%$. A qualidade dos resultados da simulações possibilita um potencial maior do conhecimento sobre os equipamentos de infusão, os quais possuem um elevado índice de eventos adversos associados.

O simulador SPODi, tem potencial para contribuir com pesquisas na área de infusoterapia, e seus desdobramentos nas decisões médicas quando da prescrição medicamentosa.

Destaca-se como expectativa de trabalho futuro, incluir no simulador SPODi outros sistemas de infusões praticados nos procedimentos de infusoterapia, tendo como premissa a concepção de uma nova versão do simulador, capaz de gerar dados tanto para entrega de medicamentos por infusões gravitacionais, como eletromecânicas.

\section{Referências}

Beaudoin, M., Kabanza, F., Nault, V., and Valiquette, L. (2016). Evaluation of a machine learning capability for a clinical decision support system to enhance antimicrobial stewardship programs. Artificial Intelligence in Medicine, 68:29-36.

Berdot, S., Gillaizeau, F., Caruba, T., Prognon, P., Durieux, P., and Sabatier, B. (2013). Drug Administration Errors in Hospital Inpatients: A Systematic Review. PLoS ONE.

Bhavasaar, M. K., Nithya, M., Praveena, R., Bhuvaneswari, N., and Kalaiselvi, T. (2016). Automated intravenous fluid monitoring and alerting system. In 2016 IEEE Technological Innovations in ICT for Agriculture and Rural Development (TIAR), pages 77-80, Chennai, India. IEEE.

Blandford, A., Furniss, D., Lyons, I., Chumbley, G., Iacovides, I., Wei, L., Cox, A., Mayer, A., Schnock, K., Bates, D. W., Dykes, P. C., Bell, H., and Franklin, B. D. (2016). Exploring the Current Landscape of Intravenous Infusion Practices and Errors (ECLIPSE): protocol for a mixed-methods observational study. BMJ Open, 6(3):e009777.

Cheng, M.-Y., Damalerio, R., Lim, R., Chen, W., Tan, K. L., Bong, C. L., and Tan, S. K. (2016). Wearable Sensor Patch for Early Extravasation Detection. In 2016 IEEE 66th Electronic Components and Technology Conference (ECTC), volume 2016Augus, pages 1632-1637, New York, USA. IEEE.

Dey, N., Ashour, A. S., Shi, F., Fong, S. J., and Tavares, J. M. R. S. (2018). Medical cyber-physical systems: A survey. Journal of Medical Systems, 42(4):74.

ECRI, I. (2017). Top 10 Health Technology Hazards for Top 10 Health Technology Hazards for 2017. Technical report, ECRI Institute Patient Safety Organization, Massachusetts, USA, Massachusetts, USA. 
Hottinger, B. M. (2018). Electrical connection of force transducers. https: / / www . hbm.com/pt/4248/conexao-eletrica-de-sensores-de-forca/. Acessado em 02/12/2018.

Kleckers, T. (2018). Important characteristics of force transducers. Technical report, HBM GmbH, Darmstadt, Germany.

MELLO, D. R. D. (2010). Farmacopeia Brasileira, volume 2. Diário Oficial da União Agência Nacional de Vigilância Sanitária (ANVISA), Brasília, Brasil.

Murphy, R. S. and Wilcox, S. J. (2010). The Link Between Intravenous Multiple Pump Flow Errors and Infusion System Mechanical Compliance. Anesthesia \& Analgesia, 110(5):1297-1302.

NBR 60601-2-24, A. B. d. N. T. (2015). Equipamento eletromédico Parte 2-24: Requisitos particulares para a segurança básica e o desempenho essencial de bombas de infusão e de controladores de infusão. $A B N T$.

NBR 8536-5, A. B. d. N. T. (2012). Equipamento de infusão para uso médico Parte 5: Equipos de infusão com bureta para uso único, alimentação por gravidade. $A B N T$.

Oliveira dos Santos, Á., Cançado Monteiro Savassi, L., and Sztajnberg, A. (2018). Proposta de uma Plataforma Crowdsourcing para Apoio à Tomada de Decisão Médica. Simpósio Brasileiro de Computação Aplicada à Saúde (SBCAS_CSBC), 18:6.

Parker, M. J., Lovich, M. A., Tsao, A. C., Deng, H., Houle, T., and Peterfreund, R. A. (2017). Novel Pump Control Technology Accelerates Drug Delivery Onset in a Model of Pediatric Drug Infusion. Anesthesia \& Analgesia, 124(4):1129-1134.

Pierce, E. T., Kumar, V., Zheng, H., and Peterfreund, R. A. (2013). Medication and Volume Delivery by Gravity-Driven Micro-Drip Intravenous Infusion: Potential Variations During “Wide-Open” Flow. Anesthesia \& Analgesia, 116(3):614-618.

Piper, R., Carr, P. J., Kelsey, L. J., Bulmer, A. C., Keogh, S., and Doyle, B. J. (2018). The mechanistic causes of peripheral intravenous catheter failure based on a parametric computational study. Scientific Reports, 8(1):3441.

Sea Electronic, M. T. C. L. (2019). China micro load cell (cz1902) - china micro load cell, body scale load cell. https://hlhsensor.en.made-in-china.com/ product/PKUQeGzJsFhp/China-Micro-Load-Cell-CZL902- .html. (Acessado em 01/07/2019).

Trigueiro, T. C. and Lira, V. V. (2014). SimTank - Um Simulador de Nível de Líquido em Tanques. Revista Principia, page 9.

Yu, D., Hsu, K.-Y., Kim, J. H., and DeLaurentis, P. (2017). Infusion pump informatics approach to quantify impact of alerts and alarms on healthcare delivery. Proceedings of the Human Factors and Ergonomics Society Annual Meeting, 61(1):681-685.

Ziuzev, A., Nesterov, K., and Mudrov, M. (2014). The software-hardware simulator of the electric drive. In 2014 16th European Conference on Power Electronics and Applications, pages 1-7, Yekaterinburg, Russia. IEEE. 